$A D-A 071 \quad 136$ UNCLASSIF IEO

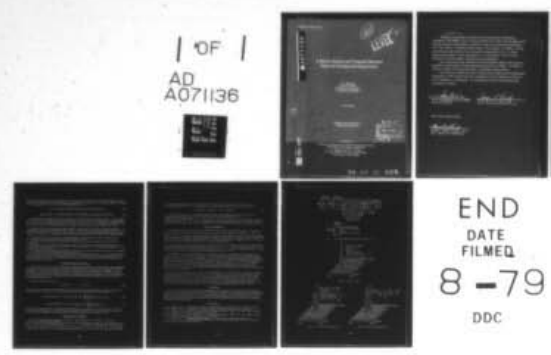

AEROSPACE CORP EL SEGUNDO CA ENGINEERING GROUP A MATRIX SPATIAL AND TEMPORAL MATCHED FILTER FOR BACKOROUND F/6 9/3 APR 79 S M MLLZER TR-0079(4901-03)-3 SAMSO-TR-79-11

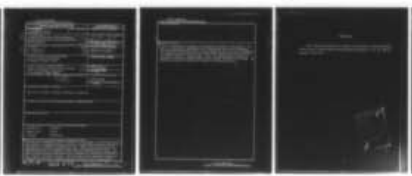

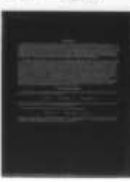
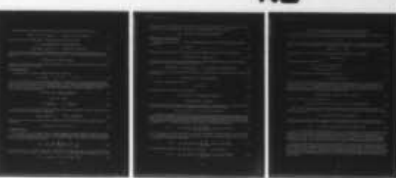

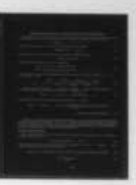




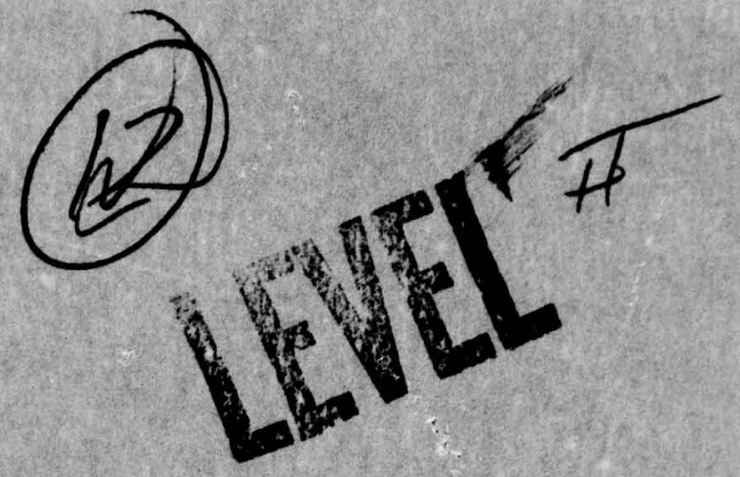

\section{A Matrix Spatial and Temporal Matched Filter for Background Suppression}

S. M. MELZER

Engineering Group

The Aerospace Corporation

El Segundo, Calif. 90245

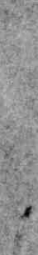

$?$
$i$
+4
+4

25 April 1979

APPROVED FOR PUBLIC RELEASE; DISTRIBUTION UNLIMITED

Prepared for

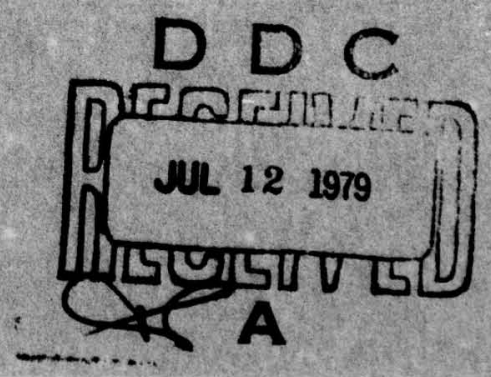

SPACE AND MISSILE SYSTEMS ORGANIZATION

AIR FORCE SYSTEMS COMMAND

Los Angeles Air Force Station

P.0. Box 92960, Worldway Postal Center

Los Angeles, Calif. 90009 
Thi final report yas submitted by The Aerospace Corporation, E1 Segundo, CA-90245, under Contract F04701-78-C-0079 with the Space and Missile Systems Organization, Deputy for Space Communications Systems, P.O. Box 92960, Worldway Postal Center, Los Angeles, CA 90009. It was reviewed and approved for The Aerospace Corporation by D. J. Griep, Engineering Group. First Lieutenant A. G. Fernandez, YCPT was the Deputy for Advanced Space Programs project engineer.

This report has been reviewed by the Information Office (OI) and is releasable to the National Technical Information Service (NTIS). At NTIS, it will be available to the general public, including foreign nations.

This technical report has been reviewed and is approved for publication. Publication of this report does not constitute Air Force approval of the report's findings or conclusions. It is published only for the exchange and stimulation of ideas.

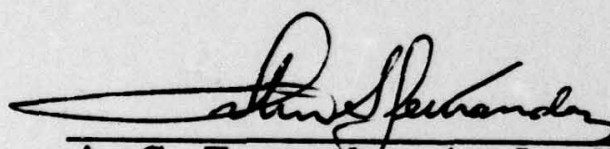

A. G. Fernandez, 1st Lt, USAF Project Engineer

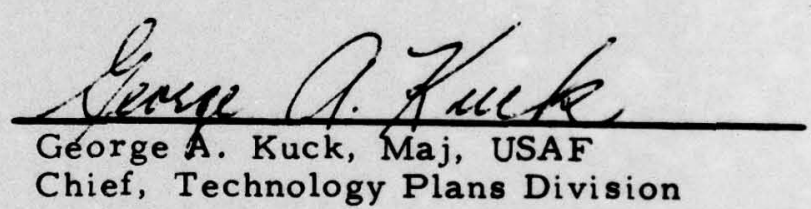

George A. Kuck, Maj, USAF
Chief, Technology Plans Division

FOR THE COMMANDER

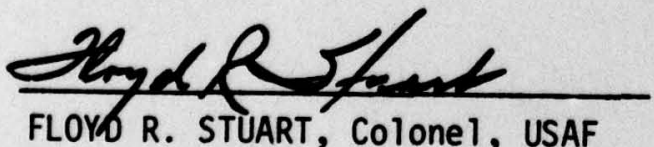

FLOYO R. STUART, Colonel, USAF

Asst. Deputy for Technology 


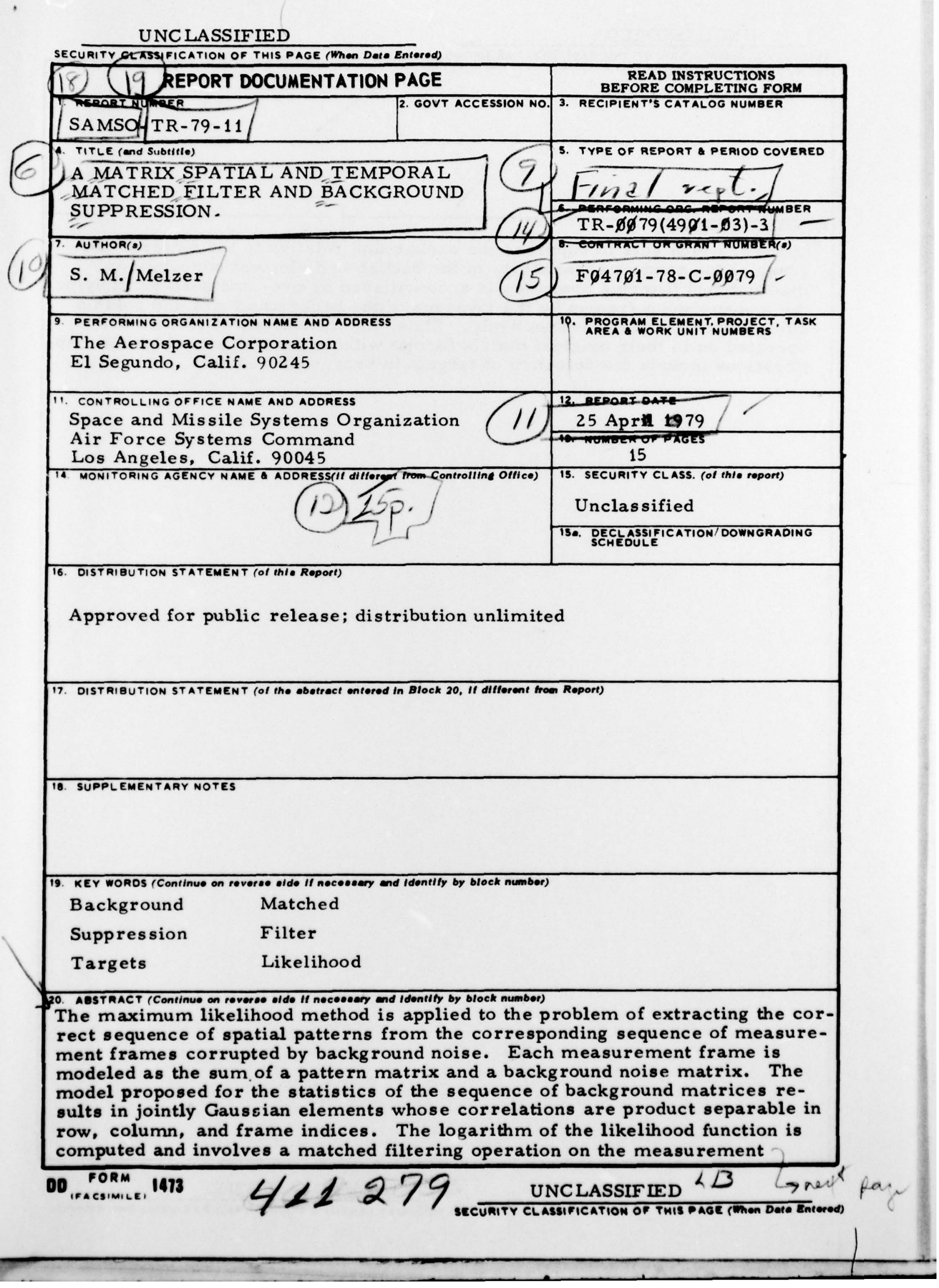




\section{UNCLASSIFIED}

SECURITY CLASSIFICATION OF THIS PAGE(Whan Date Entored)

19. KEY WORDS (Continued)

20. ABSTRACT (ContInued)

frames, which acts to suppress the background relative to the pattern. Because of the product separability in the background element correlations, this matched filtering operation is accomplished by pre- and post-multiplying the measurement frames by the inverses of the background row and column correlation matrices, respectively. Thus, the measurement frames are operated on in their original matrix format without resorting to stacking. Applications include the detection of targets in background noise.

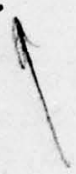




\section{PREFACE}

This report was prepared as a paper for publication in the proceedings of the Society of Photo-Optical Instrumentation Engineers, Vol. 178, Smart Sensors, April 1979.

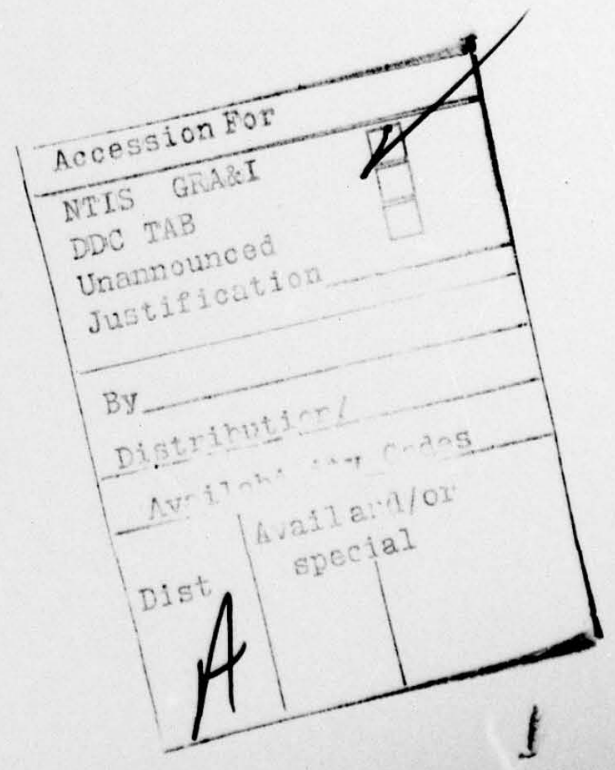


CONTENTS

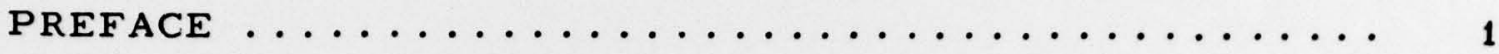

INTRODUCTION. .......................

BACKGROUND NOISE MODEL ................. 7

Transform Statistics...................... 8

Probability Density...................... 8

Multiple Frames of Background ............... 9

MAXIMUM LIKELIHOOD DETECTION OF A

PATTERN FROM A SINGLE FRAME ................ 11

MAXIMUM LIKELIHOOD DETECTION OF A SEQUENCE OF

PATTERNS FROM MULTIPLE FRAMES ............. 13

Orthogonal Pattern Sequences.................. 13

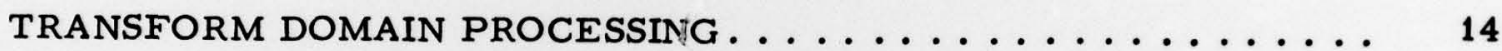

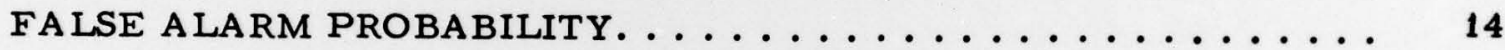

NUMERICAL EXAMPLES ..................... 15

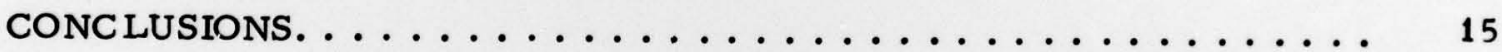

REFERENCES .......................... 15 


\section{FIGURES}

1. Moving Target Multiple Frame Processor ............ 15

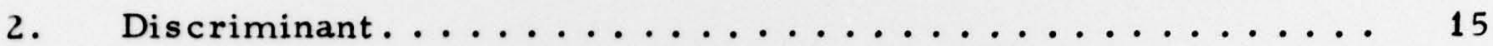

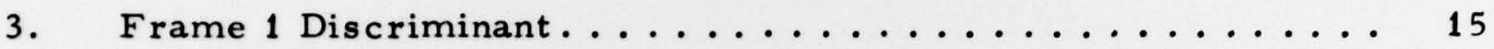

4. Frame 2 Discriminant. .................... 15 


\section{Introduction}

In digital image processing problems, the measurements are often represented by a matrix or a sequence of matrices, for example, multiple frames collected over time of a two-dimensional scene. The maximum likelihood method developed for the detection of a signal vector given a measurement vector can be applied to the detection of a signal matrix given a measurement matrix by stacking the columns (or rows) of these matrices to form "long" vectors. The resulting matched filtering operation is implemented by a left matrix multiplication on the long measurement vector requiring a large number of multiply and add computations. For real-time processing, it is desirable to avoid stacking and to operate on the measurements in their original matrix format in order to reduce the number of computations.

The paper is organized as follows: First the proposed background noise model is developed to provide the probability density functions required for the subsequent application of the maximum likelihood method. The model is an extension of that discussed in Ref. 1 for an image transform coding application. Both the cases of a single frame of background and multiple frames of background are considered. Next, the maximum likelihood method is applied to the problem of choosing the most likely pattern out of a set of possible patterns for the case of a single measurement frame. The extension is then made to the problem of choosing the most likely pattern sequence out of a set of possible pattern sequences for the case of multiple measurement frames. For the multiple frame case, the maximum likelihood method results in temporal (i.e., frame-to-frame) matched filtering, in addition to the spatial matched filtering characteristic of the single frame case. Also discussed in the paper is the alternative of transform domain processing. Finally, two numerical examples are presented relating to the detection of targets. For comparison with other authors, note that in Ref. 2 both temporal and spatial matched filtering are combined within the framework of a stacked measurement vector. In Ref. 3 results are presented for temporal matched filtering alone applied to the problem of target detection.

\section{Background Noise Model}

Background noise is modeled after extraction of its mean as an invertable left and right matrix multiplication on a zero mear stationary white matrix:

$$
\mathrm{X}=\mathrm{HWG}^{\mathrm{T}} ; \operatorname{Trace}\left(\mathrm{H}^{\mathrm{T}} \mathrm{H}\right)=\mathrm{N} ; \operatorname{Trace}\left(\mathrm{G}^{\mathrm{T}} \mathrm{G}\right)=\mathrm{M}
$$

In Eq. (1), $X=[b(k, i)]$ is an $N \times M$ matrix representing background, $H$ is an $N \times N$ matrix, $G$ is an $M \times M$ matrix, and $w=[w(k, i)]$ is an $N \times M$ stationary white matrix.

The elements of the white matrix have the second-order statistics

$$
E[w(k, i)]=0 \quad ; \quad E[w(k, i) w(r, s)]=\sigma^{2}{ }^{\delta} k, r^{\delta} i, s
$$

where $\delta_{k, r}=1$ only if $\mathrm{k}=\mathrm{r}$ and is otherwise zero. The model proposed here is an extension of the model assumed for generating the second-order statistics of random vectors, i. e., that the random vector is formed by a matrix multiplication on a stationary white vector. 
It follows from Eq. (2) that, for arbitrary $N \times N$ matrix $Q_{1}$ and arbitrary $M \times M$ matrix $Q_{2}$

$$
E\left[W^{T} Q_{1} W\right]=\operatorname{Trace}\left(Q_{1}\right) \sigma^{2} I_{M} \quad ; \quad E\left[W Q_{2} W^{T}\right]=\operatorname{Trace}\left(Q_{2}\right) \sigma^{2} I_{N}
$$

where $I_{M}$ is the $M \times M$ identity matrix. It follows from Eqs. (1) and (3) that

$$
\begin{gathered}
\sigma^{2}=\operatorname{Trace}(\mathrm{E}[\mathrm{XX} / \mathrm{NM}])=\operatorname{Trace}\left(\mathrm{E}\left[\mathrm{X}^{\mathrm{T}} \mathrm{X} / \mathrm{NM}\right]\right) \\
\mathrm{E}\left[\mathrm{XX} \mathrm{X}^{\mathrm{T}} / \mathrm{M} \sigma^{2}\right]=\mathrm{HH}^{\mathrm{T}} \triangleq \mathrm{R}_{1} \quad ; \quad \mathrm{E}\left[\mathrm{X}^{\mathrm{T}} \mathrm{X} / \mathrm{N} \sigma^{2}\right]=\mathrm{GG}^{\mathrm{T} \triangleq \mathrm{R}_{2}}
\end{gathered}
$$

where the $\mathrm{N} \times \mathrm{N}$ matrix $\mathrm{R}_{1}$ denotes the normalized row correlation matrix and the $\mathrm{M} \times \mathrm{M}$ matrix $\mathrm{R}_{2}$ the normalized column correlation matrix. It follows from Eqs. (1), (3), and (5) that the background correlation is

$$
E[b(k, i) b(r, s)]=\sigma^{2}\left[R_{1}\right]_{k, r}\left[R_{2}\right]_{i, s}
$$

where $[\cdot]_{k}, r$ denotes the $k, r$ element of the enclosed matrix. Thus, the background correlation is product separable in row and column indices.

\section{$\underline{\text { Transform Statistics }}$}

Consider next a real unitary transformation on $\mathrm{X}$ of the form:

$$
\mathrm{Z}=\mathrm{U}^{\mathrm{T}} \mathrm{XV} \quad ; \quad \mathrm{U}^{-1}=\mathrm{U}^{\mathrm{T}} \quad ; \quad \mathrm{V}^{-1}=\mathrm{V}^{\mathrm{T}}
$$

In Eq. (7), $Z=[z(n, \ell)]$ is a real $N \times M$ matrix of transform coefficients, $U$ is an $N \times N$ real unitary matrix and $\mathrm{V}$ is an $\mathrm{M} \times \mathrm{M}$ real unitary matrix. It follows that $\mathrm{X}=\mathrm{UZV}^{\mathrm{T}}$ is the inverse transformation. The individual transform coefficients are $z(n, l)=U_{n}^{T} X V_{l}$, where $U_{n}$ is the $n^{\text {th }}$ column of $U$ and $V_{l}$ is the th column of $\mathrm{V}$. It follows from Eqs. (1), (3), (5), and (7) that the transform coefficient correlation is product separable in row and column indices:

$$
\begin{array}{r}
E[z(n, l) z(r, s)]=\sigma^{2}\left(\underline{U}_{n}^{T} R_{1} \underline{U}_{r}\right)\left(\underline{V}_{\ell}^{T} R_{2} \underline{V}_{s}\right) \\
E\left[z^{2}(n, \ell)\right]=\sigma^{2} \lambda_{n}^{\beta} \beta_{l} \\
\lambda_{n} \triangleq \underline{U}_{n}^{T} R_{1} \underline{U}_{n} \quad ; \quad \beta_{l} \triangleq \underline{v}_{\ell}^{T} R_{2} \underline{V}_{\ell}
\end{array}
$$

Next, choose $U$ and $V$ such that $U^{T} R_{1} U$ and $V^{T} R_{2} V$ are diagonal matrices. Then the $\lambda_{n}$ and $\beta_{\ell}$ are the positive eigenvalues of $R_{1}$ and $R_{2}$, respectively:

$$
U^{T} R_{1} U=D_{N}\left[\lambda_{n}\right] \triangleq \lambda \quad ; \quad V^{T} R_{2} V=D_{M}\left[\beta_{l}\right] \triangleq \beta
$$

This choice of $\mathrm{U}$ and $\mathrm{V}$ is the Karhunen-Loeve transform of $\mathrm{X}$ because it gives uncorrelated transform coefficients:

$$
E[z(n, \ell) z(r, s)]=0 \text { for }(n, \ell) \neq(r, s)
$$

Probability Density

Assume that the $w(k, i)$ elements of $w$ are jointly Gaussian. It follows from Eq. (1) that the b(k, i) elements of $X$ are also jointly Gaussian. With $Z=U^{T}$ XV the Karhunen-Loeve transform, the $z(n, l)$ elements of $Z$. are mutually independent and Gaussian. The probability density of the matrix $Z$ written as a function of the transform coefficients is therefore

$$
f_{Z}(Z)=\frac{1}{A} \exp \left(-0.5 \sum_{\ell=1}^{M} \sum_{n=1}^{N} z^{2}(n, \ell) / \sigma^{2} \lambda_{n} \beta_{\ell}\right)
$$

where the constant $A=\left(2 \pi 0^{2}\right)^{N M / 2}\left(\text { Det } R_{1}\right)^{M / 2}\left(\text { Det } R_{2}\right)^{N / 2}$. But $\sum \Sigma z^{2}(n, \ell) / \lambda_{n} \beta_{\ell}=\operatorname{Trace}\left(\lambda^{-1} Z^{-1} Z^{T}\right)$ and the refore the probability density of the matrix $Z$ can be written as the following scalar function of matrices:

$$
f_{Z}(Z)=\frac{1}{A} \exp \left(-\left(0.5 / \sigma^{2}\right) \operatorname{Trace}\left(\lambda^{-1} Z \beta^{-1} Z^{T}\right)\right)
$$


From Eq. (11) $\lambda-1=U^{T} R_{1}^{-1} U$ and $\beta^{-1}=V^{T} R_{2}^{-1} V$. Thus, $\operatorname{Trace}\left(\lambda^{-1} Z \beta^{-1} Z^{T}\right)=\operatorname{Trace}\left(U^{T} R_{1}^{-1} U_{Z} V^{T} R_{2}^{-1}\right.$ $\left.V_{Z^{T}}^{T} U^{T}\right)=\operatorname{Trace}\left(R_{1}^{-1} \times R_{2}^{-1} X^{T}\right)$. Therefore, the probability density of the matrix $X$ is

$$
f_{X}(X)=\frac{1}{A} \exp \left(-\left(0.5 / \sigma^{2}\right) \operatorname{Trace}\left(R_{1}^{-1} X R_{2}^{-1} X^{T}\right)\right)
$$

Multiple Frames of Background

Consider a sequence of $X_{1} \ldots X_{L}$ of $L$ background matrices. For the $j^{\text {th }}$ frame of the sequence assume that

$$
x_{j}=H_{j} G^{T} \quad ; \quad j=1,2, \ldots, L
$$

The elements of the sequence of white matrices are assumed to have the second-order statistics

$$
E\left[w_{j}(k, i) w_{m}(r, s)\right]=\sigma^{2} \rho_{j m}{ }^{\delta} k, r^{\delta} i, s
$$

where the $\rho$ jm are elements of a positive definite $L \times L$, frame correlation matrix $p$. It follows that for arbitrary $N \times N$ matrix $Q_{1}$ and arbitrary $M \times M$ matrix $Q_{2}$

$$
E\left[w_{j}^{T} Q_{1} w_{m}\right]=\operatorname{Trace}\left(Q_{1}\right) \sigma^{2} \rho_{j m} I_{M} \quad ; \quad E\left[w_{j} Q_{2} w_{m}^{T}\right]=\operatorname{Trace}\left(Q_{2}\right) \sigma^{2} \rho_{j m} I N
$$

It follows from Eqs. (16) and (18) that the background correlation is product separable in row, column, and frame indices:

$$
E\left[b_{j}(k, i) b_{m}(r, s)\right]=\sigma^{2} \rho_{j m}\left[R_{1}\right]_{k, r}\left[R_{2}\right]_{i, s}
$$

The unitary transformation of $\mathrm{X}_{\mathrm{j}}$ is

$$
z_{j}=U^{T} X_{j} v
$$

It follows from Eqs. (10), (16), (18), and (20) that

$$
\begin{gathered}
E\left[z_{j}(n, \ell) z_{m}(r, s)\right]=\sigma^{2} \rho_{j m}\left(\underline{U}_{n}^{T} R_{1} \underline{U}_{r}\right)\left(\underline{V}_{\ell}^{T} R_{2} \underline{V}_{s}\right) \\
E\left[z_{j}(n, \ell) z_{m}(n, \ell)\right]=\sigma^{2} \rho_{j m}{ }_{n}{ }^{\beta} \ell
\end{gathered}
$$

For $U$ and $V$ chosen as the diagonalizing transforms of $R_{1}$ and $R_{2}$, respectively, (i. e., the Karhunen-Loeve transform) the transform coefficients at different position indices are uncorrelated:

$$
E\left[z_{j}(n, l) z_{m}(r, s)\right]=0 \quad \text { for }(n, l) \neq(r, s) \text { and all } j, m
$$

Assume that the $w_{j}(k, i)$ elements of $w_{j}$ are jointly Gaussian in both position and frame indices. It follows from Eq. (16) that the $b_{j}(k, i)$ elements of $X_{j}$ are also jointly Gaussian in both position and $f$ rame indi indices. With $Z_{j}=U^{T} X_{j} V$ the Karhunen-Loeve transform, the $z_{j}(n, l)$ elements of $Z_{j}$ are mutually independent in position indices and jointly Gaussian in frame indices. The joint probability density of the sequence of matrices $\mathrm{Z}_{1} \ldots \mathrm{Z}_{\mathrm{L}}$ written as a function of the transform coefficients is the refore

$$
f_{Z}\left(Z_{1} \ldots Z_{L}\right)=\frac{1}{A^{\prime}} \exp \left(-0.5 \sum_{m=1}^{L} \sum_{j=1}^{L} \sum_{\ell=1}^{M} \sum_{n=1}^{N} g_{j m} z_{j}(n, \ell) z_{m}(n, \ell) / \sigma^{2} \lambda_{n} \beta_{\ell}\right)
$$

In Eq. (24) the $g_{j m}$ are the elements of $p^{-1}$, the inverse of the frame correlation matrix, and the constant $A^{\prime}=\left(2 \pi \sigma^{2}\right)^{N M L / 2}\left(\text { Det } R_{1}\right)^{M L / 2}\left(\text { Det } R_{2}\right)^{N L / 2}(\text { Det } \rho)^{L / 2}$. This joint probability density can be written as the following scalar function of matrices:

$$
f_{Z}\left(Z_{1} \ldots Z_{L}\right)=\frac{1}{A^{\prime}} \exp \left(-\left(0.5 / \sigma^{2}\right) \sum_{m=1}^{L} \sum_{j=1}^{L} g_{j m} \operatorname{Trace}\left(\lambda^{-1} Z_{j} \beta^{-1} Z_{m}^{T}\right)\right)
$$

The joint probability density of the sequence of matrices $X_{1} \ldots X_{L}$ is therefore

$$
f_{X^{\prime}}\left(X_{1} \ldots X_{L}\right)=\frac{1}{A^{\prime}} \exp \left(-\left(0.5 / \sigma^{2}\right) \sum_{m=1}^{L} \sum_{j=1}^{L} g_{j m} \operatorname{Trace}\left(R_{1}^{-1} X_{j} R_{2}^{-1} x_{m}^{T}\right)\right)
$$




\section{Maximum Likelihood Detection of a Pattern from a Single Frame}

The problem is to choose the most likely pattern and its associated intensity from a single measurement frame. The measurement equation after extaction of the mean background is

$$
\mathrm{Y}=\mathrm{a} \mathrm{S}+\mathrm{X}
$$

where $\mathrm{Y}$ is the $\mathrm{N} \times \mathrm{M}$ measurement matrix, $\mathrm{X}$ is the background matrix, $\mathrm{S}$ is the pattern matrix, and the parameter "a" is the pattern intensity. The pattern $\mathrm{S}$ is a member of a collection of $\mathrm{K}$ possible patterns

$$
\mathrm{S} \epsilon\left[\mathrm{s}_{1}, \mathrm{~S}_{2}, \ldots . \mathrm{S}_{\mathrm{K}}\right]
$$

The parameter "a" is a member of the interval of possible intensities (or amplitudes)

$$
\text { a } \epsilon\left[a_{\min }, a_{\max }\right]
$$

Considered are the $K+1$ hypotheses

$$
\begin{aligned}
& \mathrm{H}_{0}: \mathrm{a}=0 \text { and no pattern present } \\
& \mathrm{H}_{\mathrm{i}}: \mathrm{a} \epsilon\left[\mathrm{a}_{\min },{ }_{\text {max }}\right] \text { and } \mathrm{S}=\mathrm{S}_{\mathrm{i}} \quad ; \quad \mathrm{i}=1,2, \ldots, \mathrm{K}
\end{aligned}
$$

The likelihood ratio for the $i^{\text {th }}$ hypothesis is defined by

$$
\Lambda_{\mathrm{i}} \triangleq \mathrm{f}\left(\mathrm{Y} / \mathrm{a}, \mathrm{H}_{\mathrm{i}}\right) / \mathrm{f}\left(\mathrm{Y} / \mathrm{H}_{0}\right)
$$

where $\mathrm{f}\left(\mathrm{Y} / \mathrm{a}, \mathrm{H}_{\mathrm{i}}\right)$ is the conditional probability density of $\mathrm{Y}$ given that $\mathrm{H}_{\mathrm{i}}$ is true at a specific intensity "a" and $\mathrm{f}\left(\mathrm{Y} / \mathrm{H}_{0}\right)$ is the conditional probability density of $\mathrm{Y}$ given the null hypothesis $\mathrm{H}_{0}$. It follows $\mathrm{f}$ rom Eq. (27) that $1_{i}=f_{X}\left(Y-a S_{i}\right) / f_{X}(Y)$ where $f_{X}(\cdot)$ is the probability density of the background. The most likely hypothesis is found by maximizing $\Lambda_{\mathrm{i}}$ or equivalently maximizing its logarithm. Define the ith hypothesis discriminant as the logarithm of the likelihood ratio:

$$
D\left(a, S_{i}\right) \triangleq \log _{e} \Lambda_{i}=\log _{e} f X^{\left(Y-a S_{i}\right)-\log _{e} f} X^{(Y)}
$$

Using Eq. (15) for $f_{X}(\cdot)$, the discriminant is

$$
\mathrm{D}(\mathrm{a}, \mathrm{S})=\left(0.5 / \sigma^{2}\right)\left[2 \text { a } \operatorname{Trace}\left(\mathrm{R}_{1}^{-1} \mathrm{YR}_{2}^{-1} \mathrm{~S}^{\mathrm{T}}\right)-\mathrm{a}^{2} \operatorname{Trace}\left(\mathrm{R}_{1}^{-1} \mathrm{SR}_{2}^{-1} \mathrm{~S}^{\mathrm{T}}\right)\right]
$$

Next, D(a, S) is maximized with respect to the amplitude "a" to yield an amplitude estimate â. The result is $\hat{a}=a$ for $a * \epsilon\left[a_{\min }, a_{\max }\right], \hat{a}=a_{\min }$ for $a<a_{\min }$ and $\hat{a}=a_{\max }$ for $a^{*}>a_{\max }$ with $a *$ the solution to $\partial \mathrm{D}(\mathrm{a}, \mathrm{S}) / \partial \mathrm{a}=0$ :

$$
a^{*}=\operatorname{Trace}\left(R_{1}^{-1} Y R_{2}^{-1} S^{T}\right) / T \operatorname{race}\left(R_{1}^{-1} S R_{2}^{-1} S^{T}\right)
$$

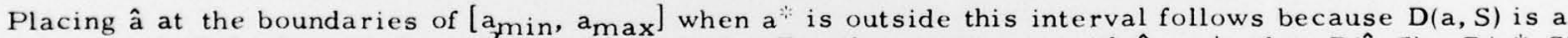
polynomial of the form $2 a C_{1}-a^{2} C_{2}$ with $C_{2}>0$. For those patterns with $a=a *$ then $D(\hat{a}, S)=D(a *, S)$ :

$$
\mathrm{D}(\mathrm{a}, \mathrm{S})=\left(0.5 / \sigma^{2}\right)\left[\operatorname{Trace}\left(\mathrm{R}_{1}^{-1} \mathrm{YR}_{2}^{-1} \mathrm{~S}^{\mathrm{T}}\right)\right]^{2} / \operatorname{Trace}\left(\mathrm{R}_{1}^{-1} \mathrm{SR}_{2}^{-1} \mathrm{~S}^{\mathrm{T}}\right)
$$

The most likely pattern maximizes $D\left(\hat{a}_{i}, S_{i}\right)$ where $E_{q} .(33)$ is used if $\hat{a}_{i}=a_{m i n}$ or $a_{m a x}$ and Eq. (35) is used if $\hat{a}_{i}=a_{i}$. Denoting the most likely pattern $\hat{S}$ then $D(\hat{a}, \hat{S})=\max D\left(\hat{a}_{i}, S_{i}\right)$. The choice is made between the most likely pattern and the null hypothesis by a threshold test. ${ }^{i}$ If $D(\hat{a}, \hat{S})>T$ accept $\hat{a}, \hat{S}$ and if $\mathrm{D}(\hat{\mathrm{a}}, \hat{\mathrm{S}}) \leq \mathrm{T}$ choose $\mathrm{H}_{0}$. The threshold is set such that the false alarm probability $\operatorname{Prob}\left[\mathrm{D}(\hat{\mathrm{a}}, \hat{\mathrm{S}})>\mathrm{T} / \mathrm{H}_{0}\right]$ is acceptably low. If the correct pattern is chosen and if $\hat{a}=a^{*}$, then $E\left[(a-\hat{a})^{2}\right]=\sigma^{2} / T_{r a c e}\left(R_{1}^{-1} S_{R}{ }^{1} S^{T}\right)$, the amplitude estimate error variance, is a measure of the degree of background suppression achieved.

For each hypothesized pattern $S$ the Trace $\left(R_{1}^{-1} Y R_{2}^{-1} S^{T}\right)$ is computed, which is a spatial matched filtering operation on the measurement matrix. This is analogous to the well-known matched filtering operation on a measurement vector (4), (5). Our case reduces to the vector case for $M=1 ;$ then $Y=y, R_{2} 1=1$, $S=\underline{s}$, and $\operatorname{Trace}\left(R_{1}^{-1} Y R_{2}^{-1} S^{T}\right)=\operatorname{Trace}\left(R_{1}^{-1} y s^{T}\right)=\underline{s}^{T} R_{1}^{-1} y$, which is the well-known form. If we had used the procedure of stacking, then $y$ would be a vector of length $N M$ and $R_{1}^{-1}$ would be a $N M \times N M$ matrix. Thus, $\mathrm{R}_{1}^{1}$ y would require (NM) ${ }^{2}$ multiplications. By contrast, the operation $R_{1}^{1} Y^{1} R_{2}^{-1}$ requires only $N M(N+M)$ multiplications. 


\section{Maximum Likelihood Detection of a Sequence of Patterns from Multiple Frames}

The problem is to choose the most likely sequence of patterns and their associated intensities from the corresponding sequence of measurement frames. The measurement equation for the $j^{\text {th }} \mathrm{frame}$ is

$$
Y(j)=a(j) S(j)+X_{j} \quad ; \quad j=1,2 \ldots L
$$

The pattern $S(j)$ is a member of a collection of $K_{j}$ possible frame $j$ patterns

$$
S(j) \epsilon\left[S_{1}(j), S_{2}(j) \ldots S_{K_{j}}(j)\right]
$$

The parameter $a(j)$ is a member of the frame $j$ interval of possible intensities

$$
a(j) \epsilon\left[a_{\min }(j), a_{\max }(j)\right]
$$

Considered for the $j^{\text {th }}$ frame are the $K_{j}+1$ hypotheses

$$
\begin{aligned}
& H_{0}(j): a(j)=0 \quad \text { and no } j^{\text {th }} \text { frame pattern } \\
& H_{i}(j): a(j) \epsilon\left[a_{\min }(j), a_{\max }(j)\right] \text { and } S(j)=S_{i}(j) ; i=1,2 \ldots K_{j}
\end{aligned}
$$

The likelihood ratio is formed assuming a particular sequence of $L$ frame hypotheses $i_{1}$, . . $i_{L}$, where $i_{j} \epsilon\left[0,1 \ldots K_{j}\right]$ :

$$
\Lambda_{i_{1}, i_{2} \ldots i_{L}} \triangleq \frac{f\left(Y(1), \ldots Y(L) / a(1), \ldots a(L) ; H_{i_{1}}(1), \ldots H_{i_{L}}(L)\right)}{f\left(Y(1), \ldots Y(L) / H_{0}(1), \ldots H_{0}(L)\right)}
$$

It follows from Eq. (36) that $\Lambda_{i_{1}}, \ldots i_{L}=f_{X}\left(Y(1)-\imath(1) S_{i_{1}}(1) \ldots . Y(L)-a(L) S_{i_{L}}(L)\right) / f_{X}(Y(1), \ldots$ $\mathrm{Y}(\mathrm{L}))$. The discriminant is defined as the logarithm of we likelihe $\mathrm{d}$ ratio:

$$
D\left(a(1), \ldots . a(L) ; S_{i_{1}}(1), \ldots S_{i_{1}}(1)\right) \triangleq \log _{e} \Lambda_{i_{1}}, \ldots i_{L}
$$

It follows from Eq. (26) for the joint probability density of $\mathrm{X}_{1} \cdots \mathrm{X}_{\mathrm{L}}$ that

$$
\begin{array}{r}
\mathrm{D}(\mathrm{a}(1), \ldots \mathrm{a}(\mathrm{L}) ; \mathrm{S}(1), \ldots \mathrm{S}(\mathrm{L}))=\left(0.5 / \sigma^{2}\right) \sum_{\mathrm{m}=1}^{\mathrm{L}} \sum_{j=1}^{\mathrm{L}} g_{j m}\left[2 a(j) \operatorname{Trace}\left(R_{1}^{-1} Y(m) R_{2}^{-1} S^{T}(j)\right)\right. \\
\left.-a(j) a(m) \operatorname{Trace}\left(R_{1}^{-1} S(m) R_{2}^{-1} S^{T}(j)\right)\right]
\end{array}
$$

In general, determination of the amplitude estimates $\hat{a}(1) \ldots . \hat{a}(L)$, which maximize the discriminant is a quadratic programming problem. There are several special pattern sequence hypotheses for which the solution is not difficult. One example is the case of a stationary scintillating pattern, which means that all frames are hypothesized to have the same pattern but possibly different intensities. A second example is the case of orthogonal pattern sequences and is discussed next.

\section{Orthogonal Pattern Sequences}

In this section, the patterns from different frames are assumed to be orthogonal. The orthogonality condition is defined here as

$$
\operatorname{Trace}\left(R_{1}^{-1} S(m) R_{2}^{-1} S^{T}(j)\right)=0 \quad \text { for } j \neq m
$$

It follows from Eq. (42) that the discriminant is frame separable, i. e., $D(a(1), \ldots \ldots(L) ; S(1), \ldots \ldots S(L))$

$=\Sigma D_{j}(a(j), S(j))$ where the $j^{\text {th }}$ frame discriminant is

$$
\begin{gathered}
D_{j}(a(j), S(j))=\left(0.5 / \sigma^{2}\right)\left[2 a(j) \operatorname{Trace}\left(R_{1}^{-1} \bar{Y}(j) R_{2}^{-1} S^{T}(j)\right)-g_{j j} a^{2}(j) \operatorname{Trace}\left(R_{1}^{-1} S(j) R_{2}^{-1} S^{T}(j)\right)\right] \\
\bar{Y}(j) \triangleq \sum_{m=1}^{L} g_{j m} Y(m)
\end{gathered}
$$


The $j^{\text {th }}$ frame discriminant is maximized with respect to the amplitude $a(j)$ to yield an amplitude estimate $\hat{a}(j)$. The result is $\hat{a}(j)=a^{*}(j)$ if $a^{*} *(j) \epsilon\left[a_{\min }(j), a_{\max }(j)\right], \hat{a}(j)=a_{\min }(j)$ if $a^{*}(j)<a_{\min }(j), a$ and $\hat{a}(j)=a_{\max }(j)$ if $a *(j)>a_{\max }(j)$ with $a *(j)$ the solution to $\partial D_{j}(a(j), S(j)) / \partial a(j)=0:$

$$
\begin{gathered}
a *(j)=\operatorname{Trace}\left(R_{1}^{-1} \bar{Y}(j) R_{2}^{-1} S^{T}(j)\right) / g_{j j} \operatorname{Trace}\left(R_{1}^{-1} S(j) R_{2}^{-1} S^{T}(j)\right) \\
D_{j}(a *(j), S(j))=\left(0.5 / \sigma^{2}\right)\left[\operatorname{Tracc}\left(R_{1}^{-1} \bar{Y}(j) R_{2}^{-1} S^{T}(j)\right)\right]^{2} / g_{j j} \operatorname{Trace}\left(R_{1}^{-1} S(j) R_{2}^{-1} S^{T}(j)\right)
\end{gathered}
$$

Denoting the most likely $j^{\text {th }}$ frame pattern $\hat{S}_{(j)}$, then $D_{j}(\hat{a}(j), \hat{S}(j))=\max _{j} D_{j}\left(\hat{a}_{i}(j), S_{i}(j)\right)$. If $D_{j}(\hat{a}(j), \hat{S}(j))>T$ accept $\hat{a}(j), \hat{S}(j)$, and if $D_{j}(\hat{a}(j), \hat{S}(j)) \leq T$ choose $H_{0}(j)$. If the correct $j$ th $i_{\text {frame pattern is chosen and if }}$ $\hat{a}(j)=a *(j)$, then the amplitude estimate error variance is $E\left[(a(j)-\hat{a}(j))^{2}\right]=\sigma^{2} / g_{j j} \operatorname{Trace}\left(R_{1}^{-1} S(j) R_{2}^{-1} S^{T}(j)\right)$. Since typically $g_{j j} \gg 1$, additional background suppression is achieved over the single frame case.

One application of the orthogonal pattern sequence case is the detection of a moving point target. For exponential row and column background correlation, two positions are orthogonal if either their row or column indices differ by 2 or greater. Shown in Fig. 1 is the moving target multiple frame processor for $a_{\min }(j)=0, a_{\max }(j)=\infty$. The determination of the target location in $\mathrm{frame} j$ involves the following steps:

1) Temporal (or frame-to-frame) matched filtering, which means that a linear combination of measurement frames is computed as given by Eq. $(45)$ to yieid $\bar{Y}(j)$.

2) Spatial matched filtering, which means that $\bar{Y}(j)$ is pre- and post-multiplied by $R_{1}^{-1}$ and $R_{2}^{-1}$, respectively.

3) Computation of the amplitude estimate matrix $A *(j)$ where each element of this $N \times M$ matrix is computed from Eq. (46) with $S(j)$ a point pattern. That is, $[A *(j)]_{k, i}=a *(j)$ computed for $[S(j)]_{k, i}=1$ and all other elements of $S(j)$ zero.

4) Computation of the discriminant matrix $D *(j)$ where each element of this $\mathrm{N} \times \mathrm{M}$ matrix is computed from Eq. (47) with $S(j)$ a point pattern as discussed in step 3 .

5) Determination of the largest $[D *(j)]_{k}$, $i$ with the corresponding $[A *(j)]_{k, i}>0$. If this largest element of $D *(j)$ exceeds the threshold $T$, then accept its indices as the most likely target position and the corresponding element of $A *(j)$ as the amplitude estimate. If the threshold is not exceeded, then decide that there is no target in frame $j$.

\section{Transform Domain Processing}

Assume that $U$ and $V$ are chosen from among the unitary transform matrices that have fast algorithms and are known to de-correlate data. Examples include the discrete cosine, Walsh-Hadamard, and Slant transforms. Assume that the particular choice of transform is close enough to the Karhupen-Loeve transform for the background frames such that $\lambda_{n}$ and $\beta_{\ell}$ parameters exist with $R_{1} \approx U \lambda U^{T}, R_{2} \approx V \beta V T$ The spatial statistics of the background frames for transform domain processing a re characterized by the $\lambda_{n}$ and $\beta_{\ell}$ parameters. These parameters would be found by appropriate statistical tests on frames of transformed backg round(1).

Define for the single frame case

$$
\mathrm{Z}_{\mathrm{Y}} \triangleq \mathrm{U}^{\mathrm{T}} \mathrm{Y} \mathrm{v} \quad ; \quad \mathrm{Z}_{\mathrm{S}}=\mathrm{U}^{\mathrm{T}} \mathrm{S} \mathrm{V}
$$

where $Z_{Y}$ and $Z_{S}$ are the $N \times M$ transforms of the measurement matrix and pattern matrix, respectively. Equations (33) - (35) apply for the determination of most likely pattern with the substitutions

$$
\begin{gathered}
\operatorname{Trace}\left(R_{1}^{-1} Y_{2}^{-1} S^{T}\right)=\operatorname{Trace}\left(\lambda^{-1} Z_{Y} \beta^{-1} Z_{S}^{T}\right)=\sum_{\ell=1}^{M} \sum_{n=1}^{N}\left[Z_{Y}\right]_{n, l}\left[Z_{S}\right]_{n, \ell} / \lambda_{n}{ }^{\beta} \ell \\
\operatorname{Trace}\left(R_{1}^{-1} S R_{2}^{-1} S^{T}\right)=\operatorname{Trace}\left(\lambda^{-1} Z_{S}{ }^{-1} Z_{S}^{T}\right)=\sum_{\ell=1}^{M} \sum_{n=1}^{N}\left[Z_{S}\right]_{n, \ell}^{2} / \lambda_{n}{ }^{\beta} \ell
\end{gathered}
$$

Define for the multiple frame, orthogonal pattern sequence case, $\bar{Z}_{Y}(j)=U T \bar{Y}(j) V$ and $Z_{S}(j)=U^{T} S(j) V$. Equations (44)-(47) apply for the determinaţion of the most likely $j$ th frame pattern with the substitutions for $\operatorname{Trace}\left(R_{1}^{-1} \bar{Y}(j) R_{2}^{-1} S^{T}(j)\right)$ and $\operatorname{Trace}\left(R_{1}^{-1} S(j) R_{2}^{-1} S^{T}(j)\right)$ analogous to $E_{q} s$. (49) and $(50)$.

\section{False Alarm Probability}

For the single frame case, if $H_{0}$ is true then $Y=X$ and $D\left(a_{i}^{*}, S_{i}\right)=0.5 \eta_{i}^{2}$ where $\eta_{i} \triangleq$ Trace $\left(R_{1}^{1} \times R_{2}^{-1} S_{i}^{T}\right) / \sigma \sqrt{\text { Trace }\left(R_{1}^{1} S_{i} R_{2}^{1} S_{i}^{T}\right)}$ is zero mean Gaussian with unity variance. A false alarm occurs if one or more of the discriminants $\mathrm{D}\left(\hat{\mathrm{a}}_{\mathrm{i}}, \mathrm{S}_{\mathrm{i}}\right) ; \mathrm{i}=1,2 \ldots \mathrm{K}$ exceeds the threshold $\mathrm{T}$ given that $\mathrm{H}_{0}$ is true. If $a_{\min }=0$ and $a_{\max }=\infty$ then a false alarm occurs if one or more of ti. $\eta_{i}$ exceeds $\sqrt{2 \mathrm{~T}}$. The probability of false alarm is therefore one minus the probability that all the $\eta_{i} \epsilon(-\infty, \sqrt[1]{2 T})$. The difficulty in making 
the calculation is that these $\eta_{i}$ are in general correlated. If the patterns $S_{i}$ are orthogonal (i.e. , $\operatorname{Trace}\left(R_{1}^{-1} S_{i} R_{2}^{-1} S_{j}^{T}\right)=0$ for $\left.i \neq j\right)$, then these $\eta_{i}$ are independent and the false alarm probability is

$$
\operatorname{Prob}\left[\mathrm{D}\left(\hat{\mathrm{a}}, \hat{\mathrm{S}}>\mathrm{T} / \mathrm{H}_{0}\right]=1-(0.5+\operatorname{erf} \sqrt{2 \mathrm{~T}})^{\mathrm{K}}\right.
$$

For the multiple frame, orthogonal pattern sequence case, if $\mathrm{H}_{0}(\mathrm{j})$ is true then $\mathrm{Y}(\mathrm{j})=\mathrm{X}_{\mathrm{j}}$ and $D_{j}\left(a_{i}(j), S_{i}(j)\right)=0.5 \eta_{i}^{2}(j)$. The random variable $\eta_{i}(j) \triangleq \operatorname{Trace}\left(R_{i}^{-1} \bar{X}_{j} R_{2}^{-1} S_{i}^{T}(j)\right) /$ $\sigma \sqrt{g_{j j} \text { Trace }\left(R_{1}^{-1} S_{i}(j) R_{2}^{-1} S_{i}^{T}(j)\right)}$ is zero mean Gaussian with unity variance where $\bar{x}_{j} \triangleq \sum_{g_{j m}} X_{m}$ is defined
analogously to Eq. (45). If $a_{\min }(j)=0$ and $a_{\max }(j)=\infty$ and if the patterns $S_{i}(j)$ are orthogonal in both $i$ and $j$ indices, then the false alarm probability for each frame is the same as $\mathrm{Eq}_{q}$. (51) with $\mathrm{K}_{\mathrm{j}}$ replacing $\mathrm{K}$.

\section{Numerical Examples}

Considered first is the detection of a point target from a single frame given that $a_{\min }=0, a_{\max }=\infty$. The normalized correlation matrices $R_{1}$ and $R_{2}$ are assumed to be exponential with $\left[R_{1}\right]_{k, i}=\rho|k-i|$ and $\left[\mathrm{R}_{2}\right]_{\mathrm{k}, \mathrm{i}}=\rho 2 \mathrm{k-i} \mid$. For computer simulation, the background is generated by $\mathrm{X}=\mathrm{HWG}^{\mathrm{T}}$ where the elements of $W$ are obtained by calls to a Gaussian number generator, $H$ is the lower triangular solution to $H H^{T}=R_{1}$ and $G$ is the lower triangular solution to $G_{G}^{T}=R_{2}$. The simulated frame of measurements is $Y=a S+X$ where $S$ is a matrix of all zeros except for a one at the target position.

The example is for a frame size $N=M=16$ with threshold $T=6$. Shown in Fig. 2 is a 3-dimensional plot of the discriminant matrix $D *$ for the case $\sigma=1, a=1, \rho_{1}=\rho_{2}=0.9$ and target position $(5,7)$. The largest element of $D$ : is at the correct position with $[\mathrm{D} *]_{5}, 7=41.9>T$. The corresponding $[A \cdots]_{5,7}=0.961$ and since this is positive it follows that the amplitude estimate is $\hat{a}=0.961$.

Considered second is the detection of a moving point target from two frames given that $a_{\min }(j)=0$, $a_{\max }(j)=\infty$ for $j=1,2$. It follows from Eqs. (36) and (45) that $\bar{Y}(1)=g_{11} a(1) S(1)+g_{12} a(2) S(2)+g_{11} X_{1}$ $+\mathrm{g}_{12} \mathrm{X}_{2}$ and $\bar{Y}(2)=\mathrm{g}_{21} \mathrm{a}(1) \mathrm{S}(1)+\mathrm{g}_{22} \mathrm{a}(2) \mathrm{S}(2)+\mathrm{g}_{21} \mathrm{X}_{1}+\mathrm{g}_{22} \mathrm{X}_{2}$. Assuming exponential correlation between frames of $\rho$ then $g_{11}=g_{22}=1 /\left(1-\rho^{2}\right)$ and $g_{12}=g_{21}=-\rho /\left(1-\rho^{2}\right)$. The background matrices $X_{1}$ and $X_{2}$ are generated from $X_{1}=\mathrm{HW}_{1} \mathrm{G}^{\mathrm{T}}$ and $\mathrm{X}_{2}=\mathrm{HW}_{2} \mathrm{G}^{\mathrm{T}}$ where $\mathrm{E}\left[\mathrm{w}_{1}(\mathrm{k}, \mathrm{i}) \mathrm{w}_{2}(\mathrm{k}, \mathrm{i})\right]=\sigma^{2} \rho$. The elements $\mathrm{w}_{2}(\mathrm{k}, \mathrm{i})$ and $w_{2}(k, i)$ are simulated by the two equations $w_{1}(k, i)=\sigma n_{1}(k, i)$ and $w_{2}(k, i)=\rho w_{1}(k, i)+\left(1-\rho^{2}\right) 1 / 2$ $\sigma n_{2}(k, i)$, respectively, where $n_{1}(k, i)$ and $n_{2}(k, i)$ are obtained by separate calls to a unity variance Gaussian number generator. The normalized correlation matrices $R_{1}$ and $R_{2}$ are assumed to be exponential, as in the first example.

The example is for frame size $N=M=16$ with threshold $T=6$. Shown in Figs. 3 and 4 are 3-dimensional plots of $\mathrm{D}(1)$ and $\mathrm{D}(2)$ for the case $\sigma=1, a(1)=a(2)=0.5, \rho_{1}=\rho_{2}=\rho=0.9$ and $\mathrm{frame} 1$ and 2 target positions $(6,12)$ and $(5,10)$, respectively. The largest element of $D *(1)$ is at the correct frame 1 position with $[\mathrm{D}(1)]_{6,12}=59.7$. The corresponding amplitude estimate is $\hat{\mathrm{a}}(1)=0.500$. The largest element of $\mathrm{D}(2)$ is at the incorrect frame 2 position of $(6,12)$. However, this position is rejected because the corresponding amplitude estimate is negative. The second largest element of $D *(2)$ is at the correct position with $[\mathrm{D}(2)]_{5,10}=49.2$. The corresponding amplitude estimate is $\hat{a}(2)=0.454$.

\section{Conclusions}

The maximum likelihood method has been applied to the problem of choosing the most likely pattern sequence from multiple measurement frames corrupted by background. By assuming that the background correlation is product separable in row, column, and frame indices, the resulting processor is a matrix spatial and temporal matched filter in which the measurement frames are processed in their original matrix format. If desired, the processing can be done in a transform domain in order to take advantage of fast transform algorithms.

\section{References}

1. Melzer, S. M., "An Image Transform Coding Algorithm Based on a Generalized Correlation Model," SPIE, Vol. 147, Applications of Digital Image Processing, San Diego, CA, Aug 1978.

2. Rauch, H. E., "Optical Discrimination for Signal Detection," SPIE, Vol. 156, Modern Utilization of Infrared Technology IV, San Diego, CA, Aug 1978.

3. Liang, A. C. "A Technique for IR Background Suppression," SPIE, Vol. 124, Modern Utilization of Inf rared Technology III, San Diego, CA, Aug 1977.

4. Pratt, W. K., Digital Image Processing, John Wiley and Sons, pp. 560, 561, 1978.

5. Root, W. L., "An Introduction to the Theory of the Detection of Signals in Noise," IEEE Proc, Vol. 58, No. 5, pp. 610-623, May 1970. 


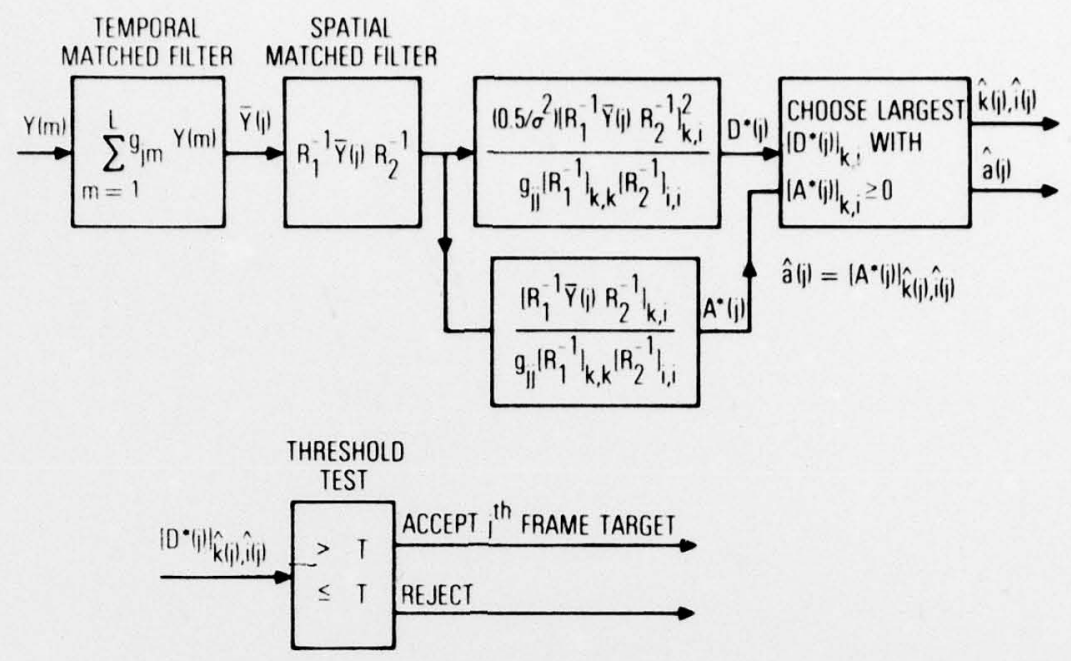

Fig. 1. Moving target multiple frame processor

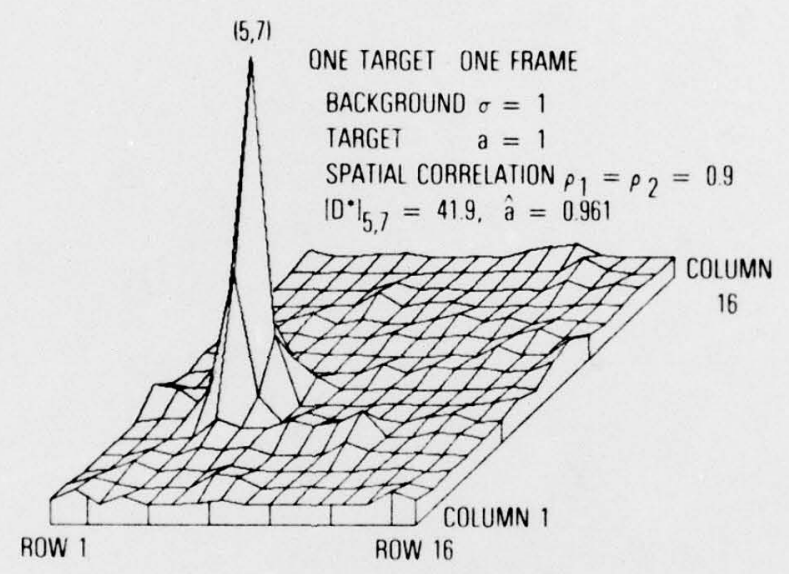

Fig. 2. Discriminant

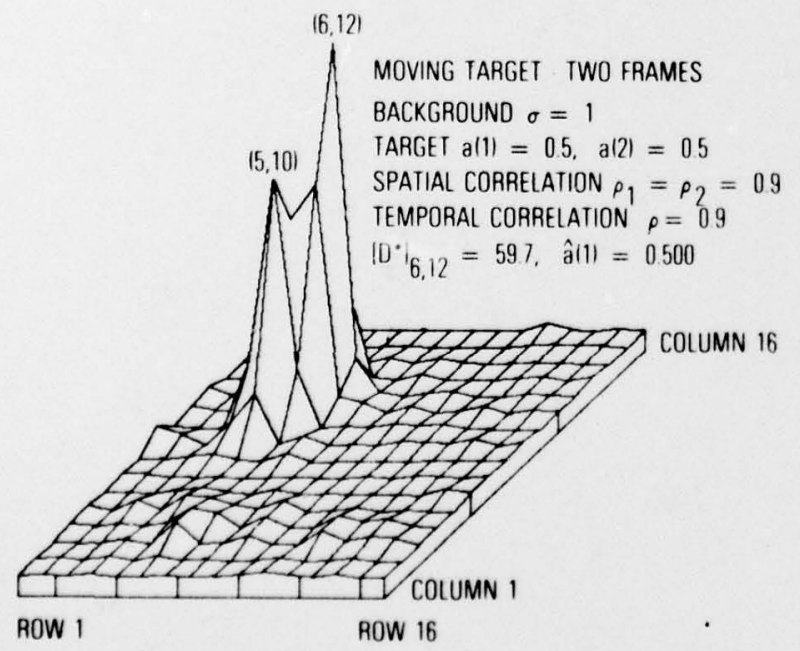

Fig. 3. Frame 1 discriminant

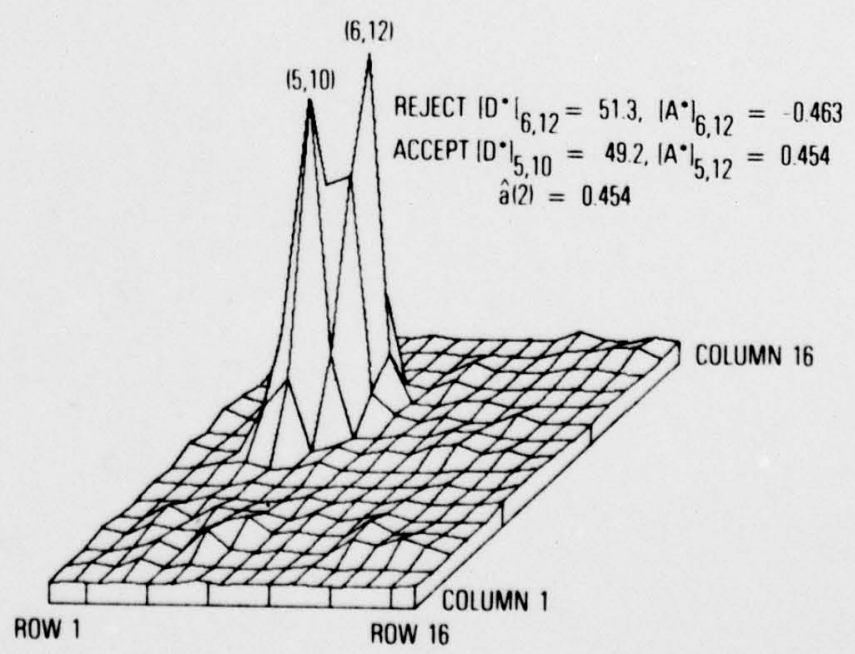

Fig. 4. Frame 2 discriminant 